\title{
Quantum Limits of Measurements and Uncertainty Principle
}

\author{
Masanao OzaWA \\ Department of Mathematics, College of General Education \\ Nagoya University, Nagoya 464, Japan
}

\section{Introduction}

In the theoretical considerations on quantum aspects of optical communications, one of the most important programs is to establish limits of measurements which are subjected to the laws of quantum mechanics in a rigorous and unified manner. In such a program, it is natural to expect that the uncertainty principle will play a central role. However, the recent controversy $[28,5,21,22]$, which arose in the field of gravitational-wave detection, on the validity of the standard quantum limit for monitoring of the free-mass position $[4,6]$ revealed a certain weakness of our understanding of the Heisenberg uncertainty principle.

Historically, Heisenberg established the uncertainty principle from his analysis of gedanken experiments for certain processes of successive measurements [10]. Thus his uncertainty principle is often stated [25, p. 239] in a form that a measurement of one variable from a conjugate pair disturbs the value of the conjugate variable no less than the order of $\hbar /$ (accuracy of the measurement). Nevertheless, we have not established a general theory of this kind of uncertainty principle, as pointed out by several authors $[3,13]$.

Our uncertainty relation in current text books was first proved by Robertson [24] by a simple mathematical reasoning with use of the Schwarz inequality. However, it is often pointed out that Robertson's uncertainty principle does not mean the Heisenberg uncertainty principle. Robertson's uncertainty principle is only concerned with state preparations as in the statement that any state preparation gives an ensemble of objects in which the product of the standard deviations of conjugate variables is greater than $\hbar / 2$ $[3,12]$.

In this paper, we shall show how the Robertson uncertainty relation gives certain intrinsic quantum limits of measurements in the most general and rigorous mathematical treatment. In Section 2, fragments from our previous work on mathematical foundations of quantum probability theory are given (see, for the detail, $[15,16,17,18,19,20]$ ). In Section 3, some basic properties of root-mean-square error of measurement, called precision, introduced in [21] is examined and, in Section 4, a general lower bound of the product of precisions arising in joint measurements of noncommuting observables is established. This result is used to give a general proof of the uncertainty relation for the joint measurements which has been found by several authors $[2,11,27,1]$. In Section 5 , we shall give a rigorous condition for holding of the standard quantum limit (SQL) for 
repeated measurements. For this purpose, we shall examine another root-mean-square error, called resolution, introduced in [21] and prove that if a measuring instruments has no larger resolution than the precision then it obeys the SQL. As shown in [21, 22, 23], we can even construct many linear models of position measurement which circumvent the above condition. In Section 6, some conclusions from the present analysis will be discussed.

\section{Foundations of quantum probability}

Let $\mathcal{H}$ be a Hilbert space. Denote by $\mathcal{L}(\mathcal{H})$ the algebra of bounded linear operators on $\mathcal{H}$, by $\tau c(\mathcal{H})$ the space of trace class operators on $\mathcal{H}$ and by $\sigma c(\mathcal{H})$ the space of HilbertSchmidt class operators on $\mathcal{H}$. A positive operator in $\tau c(\mathcal{H})$ with the unit trace is called a density operator and $\mathcal{S}(\mathcal{H})$ stands for the space of density operators on $\mathcal{H}$. Denote by $\mathcal{B}\left(\mathbf{R}^{d}\right)$ the Borel $\sigma$-field of the Euclidean space $\mathbf{R}^{d}$. A map $X: \mathcal{B}\left(\mathbf{R}^{d}\right) \rightarrow \mathcal{L}(\mathcal{H})$ is called a probability-operator-valued measure (POM) if it satisfies the following conditions (P1)-(P2):

(P1) For any sequence $\left\langle\Delta_{i} \mid i=1,2, \ldots\right\rangle$ of disjoint sets in $\mathcal{B}\left(\mathbf{R}^{d}\right)$,

$$
X\left(\bigcup_{i=1}^{\infty} \Delta_{i}\right)=\sum_{i=1}^{\infty} X\left(\Delta_{i}\right)
$$

where the sum is convergent in the weak operator topology.

(P2) $X(\mathbf{R})=1$.

A linear transformation $T: \tau c(\mathcal{H}) \rightarrow \tau c(\mathcal{H})$ is called a positive map if $T(\rho) \geq 0$ for all $\rho \in \mathcal{S}(\mathcal{H})$. We shall denote the space of all positive maps on $\tau c(\mathcal{H})$ by $\mathrm{P}(\tau c(\mathcal{H}))$. A map $\mathbf{X}: \mathcal{B}\left(\mathbf{R}^{d}\right) \rightarrow \mathrm{P}(\tau c(\mathcal{H}))$ is called an operation-valued measure if it satisfies the following conditions (O1)-(O2):

(O1) For any sequence $\left\langle\Delta_{i} \mid i=1,2, \ldots\right\rangle$ of disjoint sets in $\mathcal{B}\left(\mathbf{R}^{d}\right)$,

$$
\mathbf{X}\left(\bigcup_{i=1}^{\infty} \Delta_{i}\right)=\sum_{i=1}^{\infty} \mathbf{X}\left(\Delta_{i}\right)
$$

where the sum is convergent in the strong operator topology of $\mathrm{P}(\tau c(\mathcal{H}))$.

(O2) For any $\rho \in \tau c(\mathcal{H})$,

$$
\operatorname{Tr}\left[\mathbf{X}\left(\mathbf{R}^{d}\right) \rho\right]=\operatorname{Tr}[\rho] .
$$

An operation-valued measure $\mathbf{X}: \mathcal{B}\left(\mathbf{R}^{d}\right) \rightarrow \mathrm{P}(\tau c(\mathcal{H}))$ is called a completely positive operation-valued measure (CPOM) if it satisfies the following condition (O3):

(O3) For any $\Delta \in \mathcal{B}\left(\mathbf{R}^{d}\right), \mathbf{X}(\Delta)$ is a completely positive map on $\tau c(\mathcal{H})$, i.e.,

$$
\sum_{i, j=1}^{n}\left\langle\xi_{i}\left|\mathbf{X}(\Delta)\left(\left|\eta_{i}\right\rangle\left\langle\eta_{j}\right|\right)\right| \xi_{j}\right\rangle \geq 0
$$

for all $\Delta \in \mathcal{B}\left(\mathbf{R}^{d}\right)$ and for all finite sequences $\xi_{1}, \ldots, \xi_{n}$ and $\eta_{1}, \ldots, \eta_{n}$ in $\mathcal{H}$. 
The transpose ${ }^{t} T: \mathcal{L}(\mathcal{H}) \rightarrow \mathcal{L}(\mathcal{H})$ of $T \in \mathrm{P}(\tau c(\mathcal{H}))$ is defined by the relation

$$
\operatorname{Tr}\left[{ }^{t} T(a) \rho\right]=\operatorname{Tr}[a T(\rho)],
$$

for all $a \in \mathcal{L}(\mathcal{H})$ and $\rho \in \tau c(\mathcal{H})$. In this case, ${ }^{t} T$ is also positive in the sense that $T(a) \geq 0$ for any $a \geq 0$ in $\mathcal{L}(\mathcal{H})$. For any operation-valued measure $\mathbf{X}$, the relation

$$
\hat{\mathbf{X}}(\Delta)={ }^{t} \mathbf{X}(\Delta) 1 \quad\left(\Delta \in \mathcal{B}\left(\mathbf{R}^{d}\right)\right),
$$

determines a POM $\hat{\mathbf{X}}$, called the POM associated with $\mathbf{X}$. Conversely, any POM $X$ has at least one CPOM $\mathbf{X}$ such that $X=\hat{\mathbf{X}}$ [16, Proposition 4.1]. POM's are called "measurements" in [12] and operation-valued measures are called "instruments" in [8, 7]. Our terminology is intended to be more neutral in meanings in the physical context.

Suppose that a Hilbert space $\mathcal{H}$ is the state space of a quantum system $\mathbf{S}$. A state of $\mathbf{S}$ is a density operator on $\mathcal{H}$ and an observable of $\mathbf{S}$ is a $\mathrm{POM} A: \mathcal{B}(\mathbf{R}) \rightarrow \mathcal{L}(\mathcal{H})$ such that $A(\Delta)$ is a projection for all $\Delta \in \mathcal{B}(\mathbf{R})$. A state of the form $|\psi\rangle\langle\psi|$ for a unit vector $\psi \in \mathcal{H}$ is called a pure state and $\psi$ is called a vector state of $\mathbf{S}$. A finite set $\left\{A_{1}, \ldots, A_{n}\right\}$ of observables is called compatible if $\left[A_{i}\left(\Delta_{1}\right), A_{j}\left(\Delta_{2}\right)\right]=0$ for all $i, j=1, \ldots, n$ and $\Delta_{1}, \Delta_{2} \in \mathcal{B}(\mathbf{R})$. The joint probability distribution of a compatible set $\left\{A_{1}, \ldots, A_{n}\right\}$ of observables in a state $\rho$, denoted by $\operatorname{Pr}\left[A_{1} \in \Delta_{1}, \ldots, A_{n} \in \Delta_{n} \| \rho\right],\left(\Delta_{1}, \ldots, \Delta_{n} \in \mathcal{B}(\mathbf{R})\right)$, is given by the following Born statistical formula:

$$
\operatorname{Pr}\left[A_{1} \in \Delta_{1}, \ldots, A_{n} \in \Delta_{n} \| \rho\right]=\operatorname{Tr}\left[A_{1}\left(\Delta_{1}\right) \cdots A_{n}\left(\Delta_{n}\right) \rho\right] .
$$

The symbol $\rho$ in the left-hand-side denotes the state for which the probability distribution is determined. For pure states $\rho=|\psi\rangle\langle\psi|$, the symbol $\psi$ will be sometimes used instead of $\rho$ in this and similar expressions, and then we have

$$
\operatorname{Pr}\left[A_{1} \in \Delta_{1}, \ldots, A_{n} \in \Delta_{n} \| \psi\right]=\left\langle\psi\left|A_{1}\left(\Delta_{1}\right) \cdots A_{n}\left(\Delta_{n}\right)\right| \psi\right\rangle .
$$

By spectral theory, the relation

$$
\hat{A}=\int_{\mathbf{R}} a A(d a)
$$

sets up a one-to-one correspondence between observables $A$ and self-adjoint operators $\hat{A}$.

In this paper, by a measurement we shall mean generally an experiment described as follows. Let $\mathbf{P}$ be a quantum system, called a probe system, described by a Hilbert space $\mathcal{K}$. The system $\mathbf{P}$ is coupled to the system $\mathbf{S}$ during a finite time interval from time $t$ to $t+\tilde{\tau}$. Denote by $\hat{U}$ the unitary operator on $\mathcal{H} \otimes \mathcal{K}$ corresponding to the time evolution of the system $\mathbf{S}+\mathbf{P}$ from time $t$ to $t+\tilde{\tau}$. The time $t$ is called the time of measurement and the time $t+\tilde{\tau}$ is called the time just after measurement. At the time just after the measurement, the systems $\mathbf{S}$ and $\mathbf{P}$ are separated and in order to obtain the outcome of this experiment a compatible sequence $\left\langle M_{1}, \ldots, M_{n}\right\rangle$ of observables of the system $\mathbf{P}$ are measured by an ideal manner. The observables $M_{1}, \ldots, M_{n}$ are called the meter observables. In order to assure the reproducibility of this experiment, the probe system $\mathbf{P}$ is always prepared in a fixed state $\sigma$ at the time of measurement. Thus the physical process of a given measurement is characterized by a 4 -tuple $\mathcal{X}=\left[\mathcal{K}, \sigma, \hat{U},\left\langle\hat{M}_{1}, \ldots, \hat{M}_{n}\right\rangle\right]$, called a measurement scheme, consisting of a Hilbert space $\mathcal{K}$, a density operator $\sigma$ on $\mathcal{K}$, a unitary operator $\hat{U}$ on $\mathcal{H} \otimes \mathcal{K}$ and a compatible sequence $\left\langle\hat{M}_{1}, \ldots, \hat{M}_{n}\right\rangle$ of self-adjoint 
operators on $\mathcal{K}$. Every measurement scheme $\mathcal{X}=\left[\mathcal{K}, \sigma, \hat{U},\left\langle\hat{M}_{1}, \ldots, \hat{M}_{n}\right\rangle\right]$ determines a unique $\mathrm{CPOM} \mathbf{X}: \mathcal{B}\left(\mathbf{R}^{n}\right) \rightarrow \mathrm{P}(\tau c(\mathcal{H}))$, called the CPOM of $\mathcal{X}$, by the following relation

$$
\mathbf{X}\left(\Delta_{1} \times \cdots \times \Delta_{n}\right) \rho=\operatorname{Tr}_{\mathcal{K}}\left[\left(1 \otimes M_{1}\left(\Delta_{1}\right) \cdots M_{n}\left(\Delta_{n}\right)\right) \hat{U}(\rho \otimes \sigma) \hat{U}^{\dagger}\right],
$$

for all $\rho \in \tau c(\mathcal{H})$ and $\Delta_{1}, \ldots, \Delta_{n} \in \mathcal{B}(\mathbf{R})$, where $\operatorname{Tr}_{\mathcal{K}}$ stands for the partial trace operation of $\mathcal{K}$. Then the CPOM $\mathbf{X}$ satisfies the following Davies-Lewis postulates (DL1)-(DL2) (cf. [8]):

(DL1) Measurement probability: If the state of the system $\mathbf{S}$ at the time of measurement is $\rho$, then the probability distribution $\operatorname{Pr}[X \in \Delta \| \rho],\left(\Delta \in \mathcal{B}\left(\mathbf{R}^{n}\right)\right)$, of the outcome variable $X$ of the measurement is

$$
\operatorname{Pr}[X \in \Delta \| \rho]=\operatorname{Tr}[\mathbf{X}(\Delta) \rho]
$$

(DL2) State reduction: If the state of the system $\mathbf{S}$ at the time of measurement is $\rho$, then the measurement changes the state so that the state $\rho_{\Delta}$, at the time just after measurement, of the subensemble $\mathbf{S}_{\Delta}$ of the systems selected by the condition $X \in \Delta$ is given by

$$
\rho_{\Delta}=\frac{\mathbf{X}(\Delta) \rho}{\operatorname{Tr}[\mathbf{X}(\Delta) \rho]}
$$

for any $\Delta \in \mathcal{B}\left(\mathbf{R}^{n}\right)$ with $\operatorname{Pr}[X \in \Delta \| \rho] \neq 0$.

Given an operation-valued measure $\mathbf{X}: \mathcal{B}\left(\mathbf{R}^{n}\right) \rightarrow \mathrm{P}(\tau c(\mathcal{H}))$, any measurement scheme $\mathcal{X}=\left[\mathcal{K}, \sigma, \hat{U},\left\langle\hat{M}_{1}, \ldots, \hat{M}_{n}\right\rangle\right]$ which satisfies Eq. (2.1) is called a realization of $\mathbf{X}$. An operation-valued measure is called realizable if it has at least one realization. An importance of complete positivity for operation-valued measures is clear from the following.

Theorem 2.1. An operation-valued measure $\mathbf{X}: \mathcal{B}\left(\mathbf{R}^{n}\right) \rightarrow \mathrm{P}(\tau c(\mathcal{H}))$ is realizable if and only if it is a CPOM. In particular, every $C P O M \mathbf{X}: \mathcal{B}\left(\mathbf{R}^{n}\right) \rightarrow \mathrm{P}(\tau c(\mathcal{H})$ ) has a realization $\mathcal{X}=\left[\mathcal{K}, \sigma, \hat{U},\left\langle\hat{M}_{1}, \ldots, \hat{M}_{n}\right\rangle\right]$ such that $\sigma$ is a pure state and $\operatorname{dim}(\mathcal{H})=\operatorname{dim}(\mathcal{K})$.

For a proof, see [16, Section 5]. A consequence from the above theorem is the following version of the Naimark extension of POM's.

Corollary 2.2. For any $P O M X: \mathcal{B}\left(\mathbf{R}^{n}\right) \rightarrow \mathcal{L}(\mathcal{H})$, there exists a measurement scheme $\mathcal{X}=\left[\mathcal{K},|\phi\rangle\langle\phi|, \hat{U},\left\langle\hat{M}_{1}, \ldots \hat{M}_{n}\right\rangle\right]$ satisfying the relation

$$
X\left(\Delta_{1} \times \cdots \times \Delta_{n}\right)=V^{\dagger}\left[\hat{U}^{\dagger}\left(1 \otimes M_{1}\left(\Delta_{1}\right) \cdots M_{n}\left(\Delta_{n}\right)\right) \hat{U}\right] V
$$

for all $\Delta_{1}, \ldots, \Delta_{n} \in \mathcal{B}\left(\mathbf{R}^{n}\right)$, where $V$ is the isometry from $\mathcal{H}$ to $\mathcal{H} \otimes \mathcal{K}$ such that $V \psi=$ $\psi \otimes \phi$ for all $\psi \in \mathcal{H}$. 
A measurement scheme $\mathcal{X}$ satisfying Eq. (2.2) is called an interacting realization of a POM $X$. A definition of the non-interacting version of realizations of POM's appears in $[12$, p. 68$]$ and it should be noted that an interacting realization determines the state reduction but a non-interacting one does not.

The outcome variable $X$ of a measurement scheme $\mathcal{X}$ is generally called a quantum random variable (q.r.v.). Thus any q.r.v. $X$ has a $\mathrm{CPOM} \mathbf{X}$ which determines the probability distributions of $X$. Let $\left\langle X_{1}, \ldots, X_{n}\right\rangle$ be a finite sequence of q.r.v.s of measurement schemes $\mathcal{X}_{1}, \ldots, \mathcal{X}_{n}$ and $\left\langle\mathbf{X}_{1}, \ldots, \mathbf{X}_{n}\right\rangle$ the corresponding sequence of CPOM's. Then, from (DL1) and (DL2), the joint probability distribution $\operatorname{Pr}\left[X_{1} \in \Delta_{1}, \ldots, X_{n} \in \Delta_{n} \| \rho\right]$, $\left(\Delta_{1}, \ldots, \Delta_{n} \in \mathcal{B}\left(\mathbf{R}^{n}\right)\right)$, of $\left\langle X_{1}, \ldots, X_{n}\right\rangle$ in a state $\rho$ is given by the following Davies-Lewis formula $[8]$ :

$$
\operatorname{Pr}\left[X_{1} \in \Delta_{1}, \ldots, X_{n} \in \Delta_{n} \| \rho\right]=\operatorname{Tr}\left[\mathbf{X}_{n}\left(\Delta_{n}\right) \cdots \mathbf{X}_{1}\left(\Delta_{1}\right) \rho\right] .
$$

Let $\hat{H}$ be the Hamiltonian of the system $\mathbf{S}$ and $\hat{U}_{t}=e^{-i t \hat{H} / \hbar}$ the unitary operator of the time evolution. For the Heisenberg system state $\rho$, we shall write,

$$
\rho(t)=\alpha(t) \rho=\hat{U}_{t} \rho \hat{U}_{t}^{\dagger},
$$

for the time evolution of the states in the Schrödinger picture. Suppose that a finite sequence of measurements corresponding to a sequence $\left\langle\mathbf{X}_{1}, \ldots, \mathbf{X}_{n}\right\rangle$ of CPOM is made successively at time $(0<) t_{1}<\cdots<t_{n}$, where it is supposed that $\tilde{\tau}_{i} \ll t_{i+1}-t_{i}$ for the durations $\tilde{\tau}_{i}$ of the coupling of measurement $\mathbf{X}_{i}$. We shall denote by $X_{i}\left(t_{i}\right)$ the outcome variable of the measurement $\mathbf{X}_{i}$ at time $t_{i}$. Then the joint probability distribution of the sequence $\left\langle X_{1}\left(t_{1}\right), \ldots, X_{n}\left(t_{n}\right)\right\rangle$ of the outcomes in the state $\rho=\rho(0)$ is given by the following Wigner-Davies-Lewis formula [26, 8]:

$$
\begin{aligned}
\operatorname{Pr}[ & \left.X_{1}\left(t_{1}\right) \in \Delta_{1}, X_{2}\left(t_{2}\right) \in \Delta_{2}, \ldots, X_{n}\left(t_{n}\right) \in \Delta_{n} \| \rho\right] \\
= & \operatorname{Tr}\left[\mathbf{X}_{n}\left(\Delta_{n}\right) \alpha\left(t_{n}-t_{n-1}\right) \cdots \mathbf{X}_{2}\left(\Delta_{2}\right) \alpha\left(t_{2}-t_{1}\right) \mathbf{X}_{1}\left(\Delta_{1}\right) \alpha\left(t_{1}\right) \rho\right], \\
& \left(\Delta_{1}, \Delta_{2}, \ldots, \Delta_{n} \in \mathcal{B}(\mathbf{R})\right) .
\end{aligned}
$$

Let $\mathbf{X}: \mathcal{B}\left(\mathbf{R}^{n}\right) \rightarrow \mathrm{P}(\tau c(\mathcal{H}))$ be a $\mathrm{CPOM}$ and $\rho$ a density operator on $\mathcal{H}$. A family $\left\{\rho_{x} \mid x \in \mathbf{R}^{n}\right\}$ of density operators on $\mathcal{H}$ is called a family of posterior states for a prior state $\rho$ and a CPOM $\mathbf{X}$ if it satisfies the following conditions (PS1)-(PS2):

(PS1) The function $x \mapsto \rho_{x}$ is strongly Borel measurable.

(PS2) For any $\Delta \in \mathcal{B}\left(\mathbf{R}^{n}\right)$,

$$
\int_{\Delta} \rho_{x} \operatorname{Tr}[\mathbf{X}(d x) \rho]=\mathbf{X}(\Delta) \rho .
$$

A family of posterior states always exists for any prior state $\rho$ and it is unique in the following sense: If $\left\{\rho_{x}^{\prime} \mid x \in \mathbf{R}\right\}$ is another family of posterior states for the prior state $\rho$, then $\rho_{x}^{\prime}=\rho_{x}$ for $\operatorname{Tr}[\mathbf{X}(d x) \rho]$-almost everywhere [17]. Suppose that a measurement corresponding to $\mathbf{X}$ is made for the system $\mathbf{S}$ in a state $\rho$ at the time of measurement and that the measurement gives the outcome $X=x\left(x \in \mathbf{R}^{n}\right)$. Let $\left\{\rho_{x} \mid x \in \mathbf{R}^{n}\right\}$ be a family of posterior states for the prior state $\rho$. Then, with probability $1, \rho_{x}$ is the state of the system $\mathbf{S}$ at the time just after the measurement. 


\section{Noise of approximate measurement}

Let $\mathcal{H}$ be a Hilbert space corresponding to a quantum system $\mathbf{S}$. Let $A$ be an observable of $\mathrm{S}$. In this section, we consider a measuring instrument designed to measure the value of an observable $A$ and discuss the noise contained in outcomes from the measuring instrument. Let $X$ be a q.r.v. representing the outcome from the measuring instrument. Then the probability distribution $\operatorname{Pr}[X \in \Delta \| \rho]$ of $X$ in a state $\rho$ of the system $\mathbf{S}$ at the time of measurement is represented by a POM $\hat{\mathbf{X}}$ for some CPOM $\mathbf{X}$ satisfying

$$
\operatorname{Pr}[X \in \Delta \| \rho]=\operatorname{Tr}[\hat{\mathbf{X}}(\Delta) \rho], \quad(\Delta \in \mathcal{B}(\mathbf{R})) .
$$

For simplicity of notation, we shall write $X(\Delta)=\hat{\mathbf{X}}(\Delta),(\Delta \in \mathcal{B}(\mathbf{R}))$. Let $f$ be a real Borel function on $\mathbf{R}$. The expectation $\operatorname{Ex}[f(X) \| \rho]$ of the q.r.v. $f(X)$ in a state $\rho$ is defined by

$$
\operatorname{Ex}[f(X) \| \rho]=\int_{\mathbf{R}} f(x) \operatorname{Pr}[X \in d x \| \rho],
$$

provided the integral is convergent. Denote by $\widehat{f(X)}$ the symmetric operator defined by

$$
\begin{aligned}
& \langle\xi|\widehat{f(X)}| \xi\rangle=\int_{\mathbf{R}} f(x)\langle\xi|X(d x)| \xi\rangle, \quad(\xi \in \operatorname{dom}(\widehat{f(X)})), \\
& \operatorname{dom}(\widehat{f(X)})=\left\{\xi \in \mathcal{H} \mid \int_{\mathbf{R}} f(x)^{2}\langle\xi|X(d x)| \xi\rangle<\infty\right\} .
\end{aligned}
$$

Then we have

$$
\operatorname{Ex}[f(X) \| \psi]=\langle\psi|\widehat{f(X)}| \psi\rangle,
$$

for any vector state $\psi \in \operatorname{dom}(\widehat{f(X)})$. The variance $\operatorname{Var}[X \| \rho]$ and the standard deviation $\Delta X[\rho]$ of $X$ in a state $\rho$ are defined in the usual way, i.e.,

$$
\begin{aligned}
& \operatorname{Var}[X \| \rho]=\operatorname{Ex}\left[X^{2} \| \rho\right]-\operatorname{Ex}[X \| \rho]^{2}, \\
& \Delta X[\rho]=\operatorname{Var}[X \| \rho]^{1 / 2} .
\end{aligned}
$$

We say that a $\mathrm{POM} X$ has finite second moment in a state $\rho$ if $\operatorname{Ex}\left[X^{2} \| \rho\right]<\infty$, or equivalently, if $\Delta X[\rho]<\infty$. Let $X$ be a POM with finite second moment in $\rho$. Then we have $\operatorname{dom}(\hat{X}) \supset \operatorname{ran}(\sqrt{\rho})$ and that $\hat{X} \sqrt{\rho}$ is a Hilbert-Schmidt operator [12]. Thus, we shall write $\operatorname{Tr}\left[\hat{X}^{2} \rho\right]=\operatorname{Tr}\left[(\hat{X} \sqrt{\rho})^{\dagger} \hat{X} \sqrt{\rho}\right]$. For any POM's $X, Y$ with $\Delta X[\rho], \Delta Y[\rho]<\infty$, the expression $\operatorname{Tr}[[\hat{X}, \hat{Y}] \rho]$ is defined by

$$
\operatorname{Tr}[[\hat{X}, \hat{Y}] \rho]=\operatorname{Tr}\left[(\hat{X} \sqrt{\rho})^{\dagger} \hat{Y} \sqrt{\rho}-(\hat{Y} \sqrt{\rho})^{\dagger} \hat{X} \sqrt{\rho}\right] .
$$

By the Robertson uncertainty relation [24], for any state $\rho$ and any pair of observables $A, B$ with $\Delta A[\rho], \Delta B[\rho]<\infty$, we have

$$
\Delta A[\rho] \Delta B[\rho] \geq \frac{1}{2}|\operatorname{Tr}[[\hat{A}, \hat{B}] \rho]| .
$$

The above relation is extended to any pair of POM's by Holevo [12, p.90], i.e., for any pair of POM's $X, Y$ with $\Delta X[\rho], \Delta Y[\rho]<\infty$, we have

$$
\Delta X[\rho] \Delta Y[\rho] \geq \frac{1}{2}|\operatorname{Tr}[[\hat{X}, \hat{Y}] \rho]| .
$$


We say that a POM $X: \mathcal{B}(\mathbf{R}) \rightarrow \mathcal{L}(\mathcal{H})$ is compatible with an observable $A$ (or $A$ compatible, in short) if it satisfies the relation

$$
\left[X\left(\Delta_{1}\right), A\left(\Delta_{2}\right)\right]=0
$$

for all $\Delta_{1}, \Delta_{2} \in \mathcal{B}(\mathbf{R})$. Let $\rho$ be a state at the time of measurement. For an $A$-compatible POM $X$, the joint probability distribution $\operatorname{Pr}\left[X \in \Delta_{1}, A \in \Delta_{2} \| \rho\right]$ of $X$ and $A$ in a state $\rho$ is given by

$$
\operatorname{Pr}\left[X \in \Delta_{1}, A \in \Delta_{2} \| \rho\right]=\operatorname{Tr}\left[X\left(\Delta_{1}\right) A\left(\Delta_{2}\right) \rho\right], \quad\left(\Delta_{1}, \Delta_{2} \in \mathcal{B}(\mathbf{R})\right) .
$$

By a notational convention, for a Borel measure $\mu$ on $\mathcal{B}\left(\mathcal{R}^{2}\right)$, we shall write

$$
\iint_{\mathbf{R}^{2}} f(x, y) \nu(d x, d y)=\int_{\mathbf{R}^{2}} f(x, y) \mu(d(x, y)),
$$

where $\nu$ is the joint measure on $\mathcal{B}(\mathbf{R}) \times \mathcal{B}(\mathbf{R})$ defined by $\nu\left(\Delta_{1}, \Delta_{2}\right)=\mu\left(\Delta_{1} \times \Delta_{2}\right)$, $\left(\Delta_{1}, \Delta_{2} \in \mathcal{B}(\mathbf{R})\right)$, and we shall write $\mathrm{L}^{p}\left(\mathbf{R}^{2}, \nu(d x, d y)\right)=\mathrm{L}^{p}\left(\mathbf{R}^{2}, \mu\right)$ for the $\mathrm{L}^{p}$-space of $\mu$. We define the root-mean-square error (or precision, in short) $\epsilon[X \mid A, \rho]$ of $X$ for measurement of an observable $A$ in a state $\rho$ by the relation

$$
\epsilon[X \| A, \rho]^{2}=\iint_{\mathbf{R}^{2}}(x-a)^{2} \operatorname{Tr}[X(d x) A(d a) \rho] .
$$

Obviously, $\epsilon[X \mid A, \rho]$ represents the root-mean-square deviation of the outcome $X$ of the measurement from the outcome $A$ of the ideal measurement, when these two were made simultaneously in the state $\rho$.

Lemma 3.1. Let $\mu$ be a finite Borel measure on $\mathbf{R}^{2}$. Then the relation

$$
\iint_{\mathbf{R}^{2}}(x-y)^{2} \mu(d x \times d y)=0
$$

holds if and only if for any $\Delta_{1}, \Delta_{2} \in \mathcal{B}(\mathbf{R})$,

$$
\mu\left(\Delta_{1} \times \Delta_{2}\right)=\mu\left(\left(\Delta_{1} \cap \Delta_{2}\right) \times \mathbf{R}\right) .
$$

Proof. Suppose that Eq. (3.6) holds. Let $D=\left\{(x, y) \in \mathbf{R}^{2} \mid x=y\right\}$. Then it follows from Eq. (3.6) that $\mu\left(\mathbf{R}^{2} \backslash D\right)=0$. Then we have

$$
\begin{aligned}
\mu\left(\Delta_{1} \times \Delta_{2}\right) & \left.=\mu\left(\left(\Delta_{1} \times \Delta_{2}\right) \cap D\right)=\mu\left(\left(\Delta_{1} \cap \Delta_{2}\right) \times \mathbf{R}\right) \cap D\right) \\
& =\mu\left(\left(\Delta_{1} \cap \Delta_{2}\right) \times \mathbf{R}\right) .
\end{aligned}
$$

Conversely suppose that Eq. (3.7) holds. Then we have

$$
\begin{aligned}
\mu\left(\Delta_{1} \times \Delta_{2}\right) & =\mu\left(\left(\Delta_{1} \cap \Delta_{2}\right) \times \mathbf{R}\right) \\
& =\int_{\Delta_{1} \cap \Delta_{2}} \mu(d x \times \mathbf{R}) \\
& =\int_{\Delta_{1}} \delta_{x}\left(\Delta_{2}\right) \mu(d x \times \mathbf{R}) \\
& =\int_{\mathbf{R}} \mu(d x \times \mathbf{R}) \int_{\mathbf{R}} \chi_{\Delta_{1} \times \Delta_{2}}(x, y) \delta_{x}(d y)
\end{aligned}
$$

where $\delta_{x}$ is the Dirac measure of $x \in \mathbf{R}$. Thus we obtain

$$
\begin{aligned}
\iint_{\mathbf{R}^{2}}(x-y)^{2} \mu(d x \times d y) & =\int_{\mathbf{R}} \mu(d x \times \mathbf{R}) \int_{\mathbf{R}}(x-y)^{2} \delta_{x}(d y) \\
& =0 .
\end{aligned}
$$

$Q E D$ 
Theorem 3.2. An A-compatible POM $X$ satisfies the relation

$$
\epsilon[X \| A, \rho]=0,
$$

for all density operator $\rho$ on $\mathcal{H}$ if and only if $X=A$.

Proof. Obviously, if $X=A$ then Eq. (3.8) holds. Suppose that Eq. (3.8) holds. Let $\rho \in \mathcal{S}(\mathcal{H})$ and $\Delta \in \mathcal{B}(\mathbf{R})$. Let $\mu$ be the Borel measure on $\mathbf{R}^{2}$ such that $\mu\left(\Delta_{1} \times \Delta_{2}\right)=$ $\operatorname{Tr}\left[X\left(\Delta_{1}\right) A\left(\Delta_{2}\right) \rho\right],\left(\Delta_{1}, \Delta_{2} \in \mathcal{B}(\mathbf{R})\right)$. Then since $\epsilon[X \| A, \rho]=0$, we have $\mu(\Delta \times \mathbf{R})=$ $\mu(\mathbf{R} \times \Delta)$ from Lemma 3.1, and hence

$$
\operatorname{Tr}[A(\Delta) \rho]=\operatorname{Tr}[A(\Delta) X(\mathbf{R}) \rho]=\operatorname{Tr}[A(\mathbf{R}) X(\Delta) \rho]=\operatorname{Tr}[X(\Delta) \rho] .
$$

Since $\rho$ and $\Delta$ are arbitrary, it is concluded that $A=X$. QED

Lemma 3.3. For any A-compatible POM X, there exists a Hilbert space $\tilde{\mathcal{H}}$, an isometry $V: \mathcal{H} \rightarrow \tilde{\mathcal{H}}$ and self-adjoint operators $\tilde{X}$ and $\tilde{A}$ on $\tilde{\mathcal{H}}$ satisfying the following conditions:

(1) $[\tilde{X}, \tilde{A}]=0$ and $\tilde{A} V=V \hat{A}$.

(2) For any $\rho \in \mathcal{S}(\mathcal{H})$ and $f \in \mathrm{L}^{1}\left(\mathbf{R}^{2}, \operatorname{Tr}[X(d x) A(d a) \rho]\right)$,

$$
\iint_{\mathbf{R}^{2}} f(x, a) \operatorname{Tr}[X(d x) A(d a) \rho]=\operatorname{Tr}\left[f(\tilde{X}, \tilde{A}) V \rho V^{\dagger}\right] .
$$

Proof. Let $M: \mathcal{B}\left(\mathbf{R}^{2}\right) \rightarrow \mathcal{L}(\mathcal{H})$ be a POM such that $M\left(\Delta_{1} \times \Delta_{2}\right)=X\left(\Delta_{1}\right) A\left(\Delta_{2}\right)$, for all $\Delta_{1}, \Delta_{2} \in \mathcal{B}(\mathbf{R})$. Then, by the Naimark extension of $M$, there exist a Hilbert space $\tilde{\mathcal{H}}$, an isometry $V: \mathcal{H} \rightarrow \tilde{\mathcal{H}}$ and a projection valued measure $E: \mathcal{B}\left(\mathbf{R}^{2}\right) \rightarrow \mathcal{L}(\tilde{\mathcal{H}})$ such that $M\left(\Delta_{1} \times \Delta_{2}\right)=V^{\dagger} E\left(\Delta_{1} \times \Delta_{2}\right) V$ for all $\Delta_{1}, \Delta_{2} \in \mathcal{B}(\mathbf{R})$. Let $\tilde{X}$ and $\tilde{A}$ be self-adjoint operators on $\tilde{\mathcal{H}}$ defined by

$$
\begin{aligned}
& \tilde{X}=\int_{\mathbf{R}} x E(d x \times \mathbf{R}), \\
& \tilde{A}=\int_{\mathbf{R}} a E(\mathbf{R} \times d a) .
\end{aligned}
$$

Then the assertion follows from a straightforward verification. $Q E D$

An $A$-compatible POM $X$ is said to be unbiased if $\hat{A}=\hat{X}$; in this case, we have. $\operatorname{Ex}[A \| \rho]=\operatorname{Ex}[X \| \rho]$ for all state $\rho$ with $\Delta X[\rho]<\infty$.

Theorem 3.4. Let $X$ be an unbiased A-compatible POM. Then for any state $\rho$ with $\Delta X[\rho]<\infty$, we have

$$
\epsilon[X \| A, \rho]^{2}=\Delta X[\rho]^{2}-\Delta A[\rho]^{2} .
$$


Proof. By Lemma 3.3, we have

$$
\begin{aligned}
\epsilon[X \| A, \rho]^{2} & =\iint_{\mathbf{R}^{2}}(x-a)^{2} \operatorname{Tr}[X(d x) A(d a) \rho] \\
& =\operatorname{Tr}\left[(\tilde{X}-\tilde{A})^{2} V \rho V^{\dagger}\right] \\
& =\operatorname{Tr}\left[\tilde{X}^{2} V \rho V^{\dagger}\right]-\operatorname{Tr}\left[\tilde{A}^{2} V \rho V^{\dagger}\right] \\
& =\Delta X[\rho]^{2}-\Delta A[\rho]^{2}
\end{aligned}
$$

$Q E D$

Remark. When $X$ is $A$-compatible but $\hat{A} \neq \hat{X}$, we have

$$
\epsilon[X \mid A, \rho]^{2}=\Delta X[\rho]^{2}-\Delta \hat{X}[\rho]^{2}+\operatorname{Tr}\left[(\hat{A}-\hat{X})^{2} \rho\right]
$$

where $\Delta \hat{X}[\rho]^{2}=\operatorname{Tr}\left[\hat{X}^{2} \rho\right]-\operatorname{Tr}[\hat{X} \rho]^{2}$. It follows that $\epsilon[X \mid A, \rho]$ has a lower bound such that $\epsilon[X \mid A, \rho]^{2} \geq \operatorname{Tr}\left[(\hat{A}-\hat{X})^{2} \rho\right]$.

\section{Uncertainty principle for joint measurements}

Consider a measuring instrument with two output variables $X, Y$ designed to measure the values of observables $A, B$ of a quantum system $\mathbf{S}$ described by a Hilbert space $\mathcal{H}$. Let $M: \mathcal{B}\left(\mathbf{R}^{2}\right) \rightarrow \mathcal{L}(\mathcal{H})$ be the joint $\mathrm{POM}$ of the pair $\langle X, Y\rangle$, and $\rho$ be a state of $\mathbf{S}$ at the time of measurement. Then we have

$$
\operatorname{Pr}\left[X \in \Delta_{1}, Y \in \Delta_{2} \| \rho\right]=\operatorname{Tr}\left[M\left(\Delta_{1} \times \Delta_{2}\right) \rho\right],
$$

for all $\Delta_{1}, \Delta_{2} \in \mathcal{B}(\mathbf{R})$. Let $\left\langle M_{X}, M_{Y}\right\rangle$ be the pair of marginal POM's of $M$, i.e., $M_{X}(\Delta)=$ $M(\Delta \times \mathbf{R}), M_{Y}(\Delta)=M(\mathbf{R} \times \Delta),(\Delta \in \mathcal{B}(\mathbf{R}))$. Then $M_{X}$ and $M_{Y}$ are the POM's of q.r.v.s $X$ and $Y$, respectively, and hence it is natural to assume that $M_{X}$ is an unbiased $A$-compatible POM and that $M_{Y}$ is an unbiased $B$-compatible POM. In this case, it is known $[2,11,27,1]$ that $\Delta X[\rho]$ and $\Delta Y[\rho]$ obeys a more stringent uncertainty relation than the Robertson-Holevo relation (3.4). A general proof of this fact is given below along with the ideas in $[1]$.

A pair $\langle X, Y\rangle$ of POM's is called a coexistent pair if there is a POM $M: \mathcal{B}\left(\mathbf{R}^{2}\right) \rightarrow \mathcal{L}(\mathcal{H})$ such that $X(\Delta)=M(\Delta \times \mathbf{R}), Y(\Delta)=M(\mathbf{R} \times \Delta)$, for all $\Delta \in \mathcal{B}(\mathbf{R})$.

Theorem 4.1. Let $\hat{A}, \hat{B}$ be self-adjoint operators on a Hilbert space $\mathcal{H}$. Let $\langle X, Y\rangle$ be a coexistent pair of POM's such that $X$ is an unbiased $A$-compatible POM and $Y$ is an unbiased B-compatible POM. Then, for any state $\rho$ with $\Delta X[\rho], \Delta Y[\rho]<\infty$, we have

(1) $\epsilon[X \| A, \rho] \epsilon[Y \| B, \rho] \geq \frac{1}{2}|\operatorname{Tr}[[\hat{A}, \hat{B}] \rho]|$,

(2) $\Delta X[\rho] \Delta Y[\rho] \geq|\operatorname{Tr}[[\hat{A}, \hat{B}] \rho]|$.

Proof. For simplicity, we shall prove the case where $\rho=|\psi\rangle\langle\psi|$. Let $M: \mathcal{B}\left(\mathbf{R}^{2}\right) \rightarrow$ $\mathcal{L}(\mathcal{H})$ be a POM such that $M(\Delta \times \mathbf{R})=X(\Delta)$ and $M(\mathbf{R} \times \Delta)=Y(\Delta)$. Let $\mathcal{X}=$ 
$\left[\mathcal{K},|\phi\rangle\langle\phi|, \hat{U},\left\langle\hat{M}_{1}, \hat{M}_{2}\right\rangle\right]$ be an interacting realization of $M$. Set $X_{1}=X, X_{2}=Y, A_{1}=A$, and $A_{2}=B$. Define noise operators $\hat{N}_{i}(i=1,2)$ by the relation

$$
\hat{N}_{i}=\hat{U}^{\dagger}\left(1 \otimes \hat{M}_{i}\right) \hat{U}-\hat{A}_{i} \otimes 1 .
$$

Then we have

$$
\begin{aligned}
\left\langle\psi \otimes \phi\left|\hat{N}_{i}\right| \psi \otimes \phi\right\rangle & =\left\langle\psi \otimes \phi\left|\hat{U}^{\dagger}\left(1 \otimes \hat{M}_{i}\right) \hat{U}\right| \psi \otimes \phi\right\rangle-\left\langle\psi\left|\hat{A}_{i}\right| \psi\right\rangle \\
& =\left\langle\psi\left|\hat{X}_{i}\right| \psi\right\rangle-\left\langle\psi\left|\hat{A}_{i}\right| \psi\right\rangle \\
& =0
\end{aligned}
$$

and hence

$$
\begin{aligned}
\Delta \hat{N}_{i}[\psi \otimes \phi]^{2} & =\left\langle\psi \otimes \phi\left|\hat{N}_{i}^{2}\right| \psi \otimes \phi\right\rangle \\
& =\left\langle\psi \otimes \phi\left|\left(\hat{U}^{\dagger}\left(1 \otimes M_{i}\right) \hat{U}-\hat{A}_{i} \otimes 1\right)^{2}\right| \psi \otimes \phi\right\rangle \\
& =\left\langle\psi\left|\hat{X}_{i}^{2}\right| \psi\right\rangle-\left\langle\psi\left|\hat{A}_{i}^{2}\right| \psi\right\rangle \\
& =\epsilon\left[X_{i} \mid A_{i}, \psi\right]^{2} .
\end{aligned}
$$

On the other hand, from the relations $(i, j=1,2)$

$$
\begin{aligned}
\left\langle\psi \otimes \phi\left|\hat{U}^{\dagger}\left(1 \otimes M_{i}\right) \hat{U}\left(\hat{A}_{j} \otimes 1\right)\right| \psi \otimes \phi\right\rangle & =\left\langle\psi \otimes \phi\left|\hat{U}^{\dagger}\left(1 \otimes M_{i}\right) \hat{U}\right|\left(\hat{A}_{j} \psi\right) \otimes \phi\right\rangle \\
& =\left\langle\psi\left|\hat{A}_{i} \hat{A}_{j}\right| \psi\right\rangle,
\end{aligned}
$$

we have

$$
\left\langle\psi \otimes \phi\left|\left[\hat{N}_{1}, \hat{N}_{2}\right]\right| \psi \otimes \phi\right\rangle=\langle\psi|[\hat{A}, \hat{B}]| \psi\rangle .
$$

Thus by the Robertson uncertainty relation we have

$$
\begin{aligned}
\epsilon[X \mid A, \psi] \epsilon[Y \mid B, \psi] & =\Delta \hat{N}_{1}[\psi \otimes \phi] \Delta \hat{N}_{2}[\psi \otimes \phi] \\
& \geq \frac{1}{2}\left|\left\langle\psi \otimes \phi\left|\left[\hat{N}_{1}, \hat{N}_{2}\right]\right| \psi \otimes \phi\right\rangle\right| \\
& =\frac{1}{2}|\langle\psi|[\hat{A}, \hat{B}]| \psi\rangle| .
\end{aligned}
$$

This concludes (1). From this relation, Theorem 3.4 and the Robertson uncertainty relation, we obtain

$$
\begin{aligned}
\Delta X[\rho]^{2} \Delta Y[\rho]^{2} & =\left(\epsilon[X \mid A, \rho]^{2}+\Delta A[\rho]^{2}\right)\left(\epsilon[Y \mid B, \rho]^{2}+\Delta B[\rho]^{2}\right) \\
& \geq(\epsilon[X \mid A, \rho] \epsilon[Y \mid B, \rho]+\Delta A[\rho] \Delta B[\rho])^{2} \\
& \geq|\operatorname{Tr}[[\hat{A}, \hat{B}] \rho]|^{2} .
\end{aligned}
$$

This proves (2). QED 


\section{Standard quantum limit for repeated measurements}

Let $\mathbf{X}: \mathcal{B}(\mathbf{R}) \rightarrow \mathrm{P}(\tau c(\mathcal{H}))$ be a $\mathrm{CPOM}$ and $A$ an observable of a system $\mathbf{S}$ corresponding to $\mathcal{H}$. We define the root-mean-square scatter (or resolution, in short) $\sigma[\mathbf{X} \| A, \rho]$ of a CPOM $\mathbf{X}$ for measurement of an observable $A$ in a state $\rho$ by the relation

$$
\sigma[\mathbf{X} \| A, \rho]^{2}=\iint_{\mathbf{R}^{2}}(x-a)^{2} \operatorname{Tr}[A(d a) \mathbf{X}(d x) \rho] .
$$

Let $\left\{\rho_{x} \mid x \in \mathbf{R}\right\}$ be a family of posterior state for a prior state $\rho$ and $\mathbf{X}$. Then, we have

$$
\sigma[\mathbf{X} \| A, \rho]^{2}=\int_{\mathbf{R}} \operatorname{Tr}[\mathbf{X}(d x) \rho] \int_{\mathbf{R}}(x-a)^{2} \operatorname{Tr}\left[A(d a) \rho_{x}\right] .
$$

Theorem 5.1. Let $\hat{A}$ be a self-adjoint operator on a Hilbert space $\mathcal{H}$. Let $\mathbf{X}: \mathcal{B}(\mathbf{R}) \rightarrow$ $\mathrm{P}(\tau c(\mathcal{H}))$ be a CPOM, $\rho$ a density operator on $\mathcal{H}$ with $\Delta A[\mathbf{X}(\mathbf{R}) \rho], \Delta \hat{\mathbf{X}}[\rho]<\infty$ and $\left\{\rho_{x} \mid x \in \mathbf{R}\right\}$ a family of posterior states for $\rho$ and $\mathbf{X}$. Then we have

$$
\sigma[\mathbf{X} \| A, \rho]^{2}=\int_{\mathbf{R}} \Delta A\left[\rho_{x}\right]^{2} \operatorname{Tr}[\mathbf{X}(d x) \rho]+\int_{\mathbf{R}}\left(\operatorname{Tr}\left[\hat{A} \rho_{x}\right]-x\right)^{2} \operatorname{Tr}[\mathbf{X}(d x) \rho] .
$$

Proof. From $\Delta A[\mathbf{X}(\mathbf{R}) \rho]<\infty$, we obtain

$$
\int_{\mathbf{R}} \operatorname{Tr}\left[\hat{A}^{2} \rho_{x}\right] \operatorname{Tr}[\mathbf{X}(d x) \rho]=\operatorname{Tr}\left[\hat{A}^{2} \mathbf{X}(\mathbf{R}) \rho\right]<\infty,
$$

and hence $\operatorname{Tr}\left[\hat{A}^{2} \rho_{x}\right]<\infty, \operatorname{Tr}[\mathbf{X}(d x) \rho]$-almost everywhere. Thus the assertion follows from Eq. (5.2) and the relations

$$
\begin{aligned}
\int_{\mathbf{R}}(a-x)^{2} \operatorname{Tr}\left[A(d a) \rho_{x}\right] & =\operatorname{Tr}\left[\hat{A}^{2} \rho_{x}\right]-2 x \operatorname{Tr}\left[\hat{A} \rho_{x}\right]+x^{2} \\
& =\operatorname{Tr}\left[\hat{A}^{2} \rho_{x}\right]-\operatorname{Tr}\left[\hat{A} \rho_{x}\right]^{2}+\left(\operatorname{Tr}\left[\hat{A} \rho_{x}\right]-x\right)^{2} \\
& =\Delta A\left[\rho_{x}\right]^{2}+\left(\operatorname{Tr}\left[\hat{A} \rho_{x}\right]-x\right)^{2} .
\end{aligned}
$$

$Q E D$

Let $\mathrm{X}$ be a CPOM of a measuring instrument with one output variable $X$ designed to make an unbiased measurement of an observable $A$ of a system $\mathbf{S}$ corresponding to a Hilbert space $\mathcal{H}$. Suppose that the system $\mathbf{S}$ undergoes unitary evolution during the time $\tau$ between two identical measurements described by the CPOM X. Let $\hat{U}_{\tau}$ be the unitary operator of the time evolution of the system $\mathbf{S}$, i.e., $\hat{U}_{\tau}=e^{-i \tau \hat{H} / \hbar}$, where $\hat{H}$ is the Hamiltonian of $\mathbf{S}$. Suppose that the system $\mathbf{S}$ is in a state $\rho$ at the time of the first measurement. Then at the time just after the first measurement (say, $t=0$ ) the system is in a posterior state $\rho_{x}$ with the probability distribution $\operatorname{Pr}[X \in d x \| \rho]=\operatorname{Tr}[\mathbf{X}(d x) \rho]$. From this outcome $X=x$, the observer makes a prediction $X(\tau)=h(x)$ for the outcome of the second measurement at $t=\tau$. Then the squared uncertainty of this prediction is

$$
\begin{aligned}
\Delta[\tau, \rho, x]^{2} & =\int_{\mathbf{R}}(a-h(x))^{2} \operatorname{Pr}\left[X \in d a \| \rho_{x}(\tau)\right] \\
& =\int_{\mathbf{R}}(a-h(x))^{2} \operatorname{Tr}\left[\mathbf{X}(d a) \alpha(\tau) \rho_{x}\right] .
\end{aligned}
$$


As to determination of $h(x)$, the following mean-value-prediction strategy is naturally adopted:

$$
h(x)=\operatorname{Tr}\left[\rho_{x} \hat{A}(\tau)\right]
$$

where

$$
\hat{A}(\tau)=\hat{U}_{\tau}^{\dagger} \hat{A}(0) \hat{U}_{\tau}
$$

The predictive uncertainty $\Delta[\tau, \rho]$ of this repeated measurement with the prior state $\rho$ and the time duration $\tau$ is defined as the root-mean square of $\Delta[\tau, \rho, x]$ over all outcomes $X=x$ of the first measurement, i.e.,

$$
\begin{aligned}
\Delta[\tau, \rho]^{2} & =\int \Delta[\tau, \rho, x]^{2} \operatorname{Pr}[X \in d x \| \rho] \\
& =\iint_{\mathbf{R}^{2}}(a-h(x))^{2} \operatorname{Tr}[\mathbf{X}(d a) \alpha(\tau) \mathbf{X}(d x) \rho] .
\end{aligned}
$$

Theorem 5.2. Let $\mathbf{X}: \mathcal{B}(\mathbf{R}) \rightarrow \mathrm{P}(\tau c(\mathcal{H}))$ be an unbiased A-compatible CPOM and $\rho$ a density operator on $\mathcal{H}$ with $\Delta A(0)[\mathbf{X}(\mathbf{R}) \rho], \Delta A(\tau)[\mathbf{X}(\mathbf{R}) \rho]<\infty$. If the relation

$$
\sigma[\mathbf{X} \| A, \rho] \leq \epsilon[\hat{\mathbf{X}} \| A, \alpha(\tau) \mathbf{X}(\mathbf{R}) \rho]
$$

holds then we have

$$
\Delta[\tau, \rho]^{2} \geq|\operatorname{Tr}[[\hat{A}(0), \hat{A}(\tau)] \mathbf{X}(\mathbf{R}) \rho]| .
$$

Proof. From Eqs. (5.3)-(5.5) and Theorem 3.4,

$$
\begin{aligned}
\Delta[\tau, \rho, x]^{2} & =\Delta X\left[\alpha(\tau) \rho_{x}\right]^{2} \\
& =\epsilon\left[X \| \alpha(\tau) \rho_{x}\right]^{2}+\Delta A(\tau)\left[\rho_{x}\right]^{2},
\end{aligned}
$$

and hence by Theorem 5.1 and the Robertson uncertainty principle,

$$
\begin{aligned}
& \Delta[\tau, \rho]^{2}=\int_{\mathbf{R}} \epsilon\left[\mathbf{X} \| \alpha(\tau) \rho_{x}\right]^{2}+\Delta A(\tau)\left[\rho_{x}\right]^{2} \operatorname{Tr}[\mathbf{X}(d x) \rho] \\
&=\epsilon[\hat{\mathbf{X}} \| A, \alpha(\tau) \mathbf{X}(\mathbf{R}) \rho]^{2}+\int_{\mathbf{R}} \Delta A(\tau)\left[\rho_{x}\right]^{2} \operatorname{Tr}[\mathbf{X}(d x) \rho] \\
& \geq \sigma[\mathbf{X} \| A, \rho]^{2}+\int_{\mathbf{R}} \Delta A(\tau)\left[\rho_{x}\right]^{2} \operatorname{Tr}[\mathbf{X}(d x) \rho] \\
& \geq \int_{\mathbf{R}} \Delta A(0)\left[\rho_{x}\right]^{2}+\Delta A(\tau)\left[\rho_{x}\right]^{2} \operatorname{Tr}[\mathbf{X}(d x) \rho] \\
& \geq \int_{\mathbf{R}} 2 \Delta A(0)\left[\rho_{x}\right] \Delta A(\tau)\left[\rho_{x}\right] \operatorname{Tr}[\mathbf{X}(d x) \rho] \\
& \geq \int_{\mathbf{R}}\left|\operatorname{Tr}\left[[\hat{A}(0), \hat{A}(\tau)] \rho_{x}\right]\right| \operatorname{Tr}[\mathbf{X}(d x) \rho] \\
& \geq|\operatorname{Tr}[[\hat{A}(0), \hat{A}(\tau)] \mathbf{X}(\mathbf{R}) \rho]|
\end{aligned}
$$

$Q E D$ 
The bound (5.8) is called the standard quantum limit (SQL) for repeated measurements with interval $\tau$ of an observable $A$. For the case where $A$ is the position observable $x$ of a free-mass $m$, relation (5.8) is reduced to the relation

$$
\Delta[\tau, \rho]^{2} \geq \frac{\hbar \tau}{m}
$$

which was posed in $[4,6]$ and the validity of this standard quantum limit was the subject of a long controversy $[28,5,14,21]$. By the above theorem, any measuring instrument which beats the SQL must have the resolution lager than the precision. In [28], Yuen pointed out a flaw in the original derivation of the SQL (5.9) and proposed an idea of using contractive states to beat the SQL. A model which clears the above condition and beats the SQL was successfully constructed in our previous work [21, 22] as a realization of Gordon-Louisell measurement $\{|\mu \nu a \omega\rangle\langle a|\}[9]$, where $|\mu \nu a \omega\rangle$ is a contractive state and $|a\rangle$ is a position eigenstate. Many linear-coupling models of position measurements which violates condition (5.7) are constructed in [23].

\section{Concluding remarks}

We have discussed quantum mechanical limitations on joint measurements and repeated measurements of a single object. It is shown that the uncertainty principle for joint measurements of noncommuting observables holds generally with a more stringent limit than the one usually supposed by the Robertson uncertainty relation. On the other hand, the SQL, which is also usually supposed from the Robertson uncertain relation, for repeated measurements of a single observable does not generally hold unless a certain additional condition is satisfied. The difference between these two problem is clear from the difference between those two uncertainties defined by Eq. (3.5) and Eq. (5.6) for which the optimizations are required. The crucial point is that in the latter problem we can use the result of the first measurement to predict the result of the second and hence the prediction can be based on posterior probability. However, in the problem of joint measurements, we are required to predict two outcomes only from prior probability given by the prior state. Thus we can circumvent the uncertainty principle in the problem of repeated measurements, when the measurement changes the prior state to the posterior state which has deterministic information about the future value of the observable to be measured. Of course, this future value must be significantly uncertain, if the prior state is of deterministic information about the present value and the measurement is not carried out. However, some measurement can give this present value precisely and further leaves the object in a state with deterministic information about the future value. Thus monitoring a mass in this way can give a precise information about classical force which drives the mass.

The author wishes to thank Professor Horace P. Yuen for hospitality at Northwestern University and Professor Roy J. Glauber for hospitality at Harvard University during his leave in 1988-1990. This work is supported in part by Hamamatsu Photonics K. K. 


\section{References}

[1] E. Arthurs and M. S. Goodman. Quantum correlations - a generalized Heisenberg uncertainty relation. Phys. Rev. Lett., 60, 2447-2449, (1988).

[2] E. Arthurs and J. L. Kelly. On the simultaneous measurement of a pair of conjugate observables. Bell. Syst. Tech. J., 44, 725-729, (1965).

[3] L. E. Ballentine. The statistical interpretation of quantum mechanics. Rev. Mod. Phys., 42, 358-381, (1970).

[4] V. B. Braginsky and Yu. I. Vorontsov. Quantum-mechanical limitations in macroscopic experiments and contemporary experimental technique. Uspehi Fiz. Nauk, 114, 41-53, (1974). [Sov. Phys. Usp. 17, 644 (1975)].

[5] C. M. Caves. Defense of the standard quantum limit for free-mass position. Phys. Rev. Lett., 54, 2465-2468, (1985).

[6] C. M. Caves, K. S. Throne, R. W. P. Drever, V. D. Sandberg, and M. Zimmermann. On the measurement of a weak classical force coupled to a quantum-mechanical oscillator. I. Issues of principle. Rev. Mod. Phys, 52, 341, (1980).

[7] E. B. Davies. Quantum Theory of Open Systems. Academic Press, London, 1976.

[8] E. B. Davies and J. T. Lewis. An operational approach to quantum probability. Commun. Math. Phys., 17, 239-260, (1970).

[9] J. P. Gordon and W. H. Louisell. Simultaneous measurement of noncommuting observables. In J. L. Kelly, B. Lax, and P. E. Tannenwald, editors, Physics of Quantum Electronics, pages 833-840, McGraw-Hill, New York, 1966.

[10] W. Heisenberg. The physical content of quantum kinematics and mechanics. In J. A. Wheeler and W. H. Zurek, editors, Quantum Theory and Measurement, pages 62-84, Princeton UP, Princeton, NJ, 1983. [translation into English by J. A. Wheeler and W. H. Zurek, 1981].

[11] A. S. Holevo. Statistical problems in quantum physics. In Lecture Notes in Math. 330, pages 104-119, Springer-Verlag, Berlin, 1973.

[12] A. S. Holevo. Probabilistic and Statistical Aspects of Quantum Theory. NorthHolland, Amsterdam, 1982.

[13] H. Martens and W. M. de Muynck. The inaccuracy principle. Found. Phys., 20, 357-380, (1990).

[14] W. -T. Ni. Quantum measurements and the standard quantum limit. Phys. Rev. A, 33, 2225-2229, (1986).

[15] M. Ozawa. Conditional expectation and repeated measurement of continuous quantum observables. In Lecture Notes in Math. 1021, pages 518-525, Springer-Verlag, Berlin, 1983.

[16] M. Ozawa. Quantum measuring processes of continuous observables. J. Math. Phys., 25, 79-87, (1984).

[17] M. Ozawa. Conditional probability and a posteriori states in quantum mechanics. Publ. RIMS, Kyoto Univ., 21, 279-295, (1985). 
[18] M. Ozawa. Concepts of conditional expectations in quantum theory. J. Math. Phys., 26, 1948-1955, (1985).

[19] M. Ozawa. On information gain by quantum measurements of continuous observables. J. Math. Phys., 27, 759-763, (1986).

[20] M. Ozawa. Measuring processes and repeatability hypothesis. In Lecture Notes in Math. 1299, pages 412-421, Springer-Verlag, Berlin, 1987.

[21] M. Ozawa. Measurement breaking the standard quantum limit for free-mass position. Phys. Rev. Lett., 60, 385-388, (1988).

[22] M. Ozawa. Realization of measurement and the standard quantum limit. In P. Tombesi and E. R. Pike, editors, Squeezed and Nonclassical Light, pages 263-286, Plenum, New York, 1989.

[23] M. Ozawa. Quantum mechanical models of position measurements. Phys. Rev. A, 41, 1735-1737, (1990).

[24] H. P. Robertson. The uncertainty principle. Phys. Rev., 34(1), 163-164, (1929).

[25] J. von Neumann. Mathematical Foundations of Quantum Mechanics. Princeton UP, Princeton, NJ, 1955.

[26] E. P. Wigner. The problem of measurement. Am. J. Phys., 31, 6-15, (1963).

[27] H. P. Yuen. Generalized quantum measurements and approximate simultaneous measurements of noncommuting observables. Phys. Lett. A, 91, 101-104, (1982).

[28] H. P. Yuen. Contractive states and the standard quantum limit for monitoring freemass positions. Phys. Rev. Lett, 51, 719-722, (1983). 